\title{
2-S11-2 Symposium
}

\section{Identification of biomarkers for taxanes-induced peripheral neuropathy and search for new therapies by drag-repositioning}

\author{
Satoshi Imai ${ }^{1}$, Takayuki Nakagawa ${ }^{1}, K^{2}$ Kazuo Matsubara ${ }^{1}$
}

${ }^{I}$ Dept. Clin. Pharmacol. Therap., Kyoto Univ. Hosp.

Chemotherapy-induced peripheral neuropathy (CIPN) is a frequent side effect of taxanes. Because of the as yet poor understanding of the mechanism underlying CIPN pathogenesis, there is no indicator for objective diagnosis like a biomarker. In addition, treatment options for CIPN remain largely unsatisfactory. Previous our study demonstrated that paclitaxel preferentially impair myelin-forming Schwann cells, and consequently induce dedifferentiation and demyelination of Schwann cells. Recently, in a paclitaxel CIPN mouse model, we found that an inflammatory factor is released from dedifferentiated Schwann cells in the mouse sciatic nerve into the blood, highly correlated with the onset of pain hypersensitivity. In this presentation, I will introduce the usefulness of this inflammatory factor as a biomarker for the progression of CIPN.

On the other hand, considering our previous findings, it seems that some drugs, which induce differentiation of Schwann cells and supply newly formed mature Schwann cells at sites of demyelinated lesions, may be a new therapy for CIPN. Now, I am promoting therapeutic drug screening for CIPN based on this concept, and I will talk about a part of our results in this presentation. 